Nadia von Benzon: n.r.vonbenzon@lancaster.ac.uk

Lancaster Environment Centre, University of Lancaster

\title{
Informed consent and secondary data: reflections on the use of mothers' blogs in social media research
}

\begin{abstract}
This paper seeks to extend debate on the use of blogs as qualitative data, specifically focusing on the issue of consent in research that uses publicly-available but personal content. Typically the argument has been made for the need to protect individual writers and engage with these documents in a considered manner that recognises the inherent vulnerability of amateur authors. This paper will argue that a framing of these writers that foregrounds their potential vulnerability, naivety and even ignorance, is in fact highly paternalistic, and therefore counter to an ethical approach to research. In seeking to protect authors from harm we deny their agency and diminish the act of online publication. Further, this approach, prevalent in academia, is at odds with the legal position, and arguably, the weight of social understanding. A reframing of amateur online authors as informed agents not only increases the accessibility of this data to researchers and subsequently policy makers, but also alludes to a need to credit the contribution of authors without the need to seek permission. Ultimately, this paper argues for a presumption of accountability, unless otherwise proven, and an approach to the use of self-published online material that mirrors the use of other sorts of secondary data with perceived 'professional' authors, such as magazine articles, newspaper columns or organizational literature.
\end{abstract}

\section{Keywords}

Ethics, informed consent, social media, blogs, vulnerability

\section{Introduction}

This paper seeks to contribute to social science discourse concerning blogs as data, as part of a far broader engagement with digital media, now well embedded in geographical discourse (Kitchin, 1998; Madge and O'Connor, 2006; Valentine, 2006; Thomson and Cupples, 2008; Davidson, 2008; Kinsley, 2014; Longhurst, 2016;). Thus far, a focus on the use of blogs as secondary data has not been a key geographical concern, despite decade-old calls for such (Madge, 2007; for exceptions see Chan, 2008; Dwyer and Davies, 2010). Drawing on the author's recent experience researching home education blogs, this paper seeks to reflect on key challenges presented by blogs as a source of secondary data; consent and confidentiality. Whilst blogs, and other sorts of fluid, social media publications are not new phenomenon, their popularity continues to grow as global access to the internet increases, with modes of communication evolving to a dominance of image and video sharing platforms such as Instagram and YouTube (We Are Social, 2017). Traditionally text-based modes of networking, such as Facebook, have evolved alongside, allowing users to share visual images including live streaming, blurring the definitions of exactly what a 'blog' might be. With online discussion space available for public use for over 30 years, online material has the potential to provide rich and varied data reflecting both public and expert opinion, and often interaction between the two. 
This paper briefly considers the ethical potential and limitations of engaging with blog data as a source of secondary data for qualitative data analysis, an endeavour still in its relative youth (Dwyer and Davies, 2010). Initially the paper explores the landscape and value of blogs, focusing on their use as a reflection on experiences of motherhood. This discussion illustrates the ways in which a participant group, not typically constructed as 'powerful', might be understood as competent, independent and cognizant producers of knowledge. Drawing on significant themes in the literature, and reflecting on the author's own experience, the paper focuses on the ethics of using blogs as data, and particularly the positioning of blog authors (following Snee, 2013), and their rights and responsibilities. This paper argues that understanding blog authors as agential actors rather than naive participants has potential ramifications for constructing contributors to research more broadly, particularly following Madge (2007) in viewing ethics as 'process' rather than bureaucracy.

\section{Literature review}

\section{Blogging Mothers}

There is a great deal published on the topic of women's use of the internet as a source of information and support on issues of parenting (Johnson, 2015; McDaniel et al., 2012; DeChoudhury et al., 2013). The need to seek advice and community from face-less online individuals is typically attributed a dearth of real-life support and the escalating pressure of contemporary society to be a 'good mother' (Choi et al., 2005; Orton-Johnson, 2017). Johnson (2015) suggests that the internet might provide an 'intimate mothering public' of 'surreptitious support' - in other words, a space where discussions of normally private affairs, or of 'messy' 'hidden truth' is possible. Others have found that mothers feel pressure to present themselves online in a way that maintains an ideal image of motherhood (Bartholomew et al., 2012; Jane and Dworkin, 2014), and as such, reproduce oppressive social discourse (OrtonJohnson, 2017; Madge and O'Connor, 2005; Dawson, 2016). However, mothers are well aware that others' online profiles are offering just one side of a parenting story (Dawson, 2016).

Madge and O'Connor (2005) offer an empowering analysis of this phenomenon, considering online spaces to be performative arenas in which mothers are able to 'try out' different identities (see also Crooks, 2006; Davidson, 2008). Research has demonstrated the way in which blogs might challenge mainstream representations of motherhood (Lopez, 2009), for example by exploring the way in which online forums such as Mumsnet seek to subvert notions of 'good motherhood' through honest and frank discussion of parenting challenges and play or humour (Pederson and Smithson, 2013; Mackenzie, 2017). Orton-Johnson (2017: 1) goes as far as to argue that online discourse subverting normative constructions of motherhood can 'collapse social contexts in important and interesting ways', creating what Fraser (1990) refers to as 'subaltern counterpublics'. However, it's important to recognize that access to these social networks and online platforms is not equitable, and there exists a digital divide between mothers (Cline and Haynes, 2001); mothers who engage with other mothers online are broadly white, educated, middle class and heterosexual (Madge and O'Connor, 2006; Hine, 2015). Many of the top 10 blogging 'moms' identified by Laird (2012) are also authors of books, present television programmes, or write journalistic contributions to newspapers. As such, the reach of these women goes beyond the blogs that they author.

\section{Social media and blogs as method}

McGannon et al. (2017:127) argue that: 'blogs are advantageous to study because they offer researchers an unobtrusive method to gain access to naturalistic narratives which participants use to construct their identities in a spontaneously generated format'. Qualitative social scientists typically understand the researcher's role as one of coproduction of research data as qualitative data is created in dialogue between two independent actors. Blog material therefore can be considered to be free of the sort of direct influence that might be asserted when data is generated through interaction between people (Gray, 2009). However, whilst blog entries might be considered 'naturalistic' and 'spontaneous' it is important that they are still recognised as performance (Dawson, 2016). Blog posts will be influenced by attempts to construct identity, and to manipulate others' perception of the authors' identity, and will 
be seeped with narrative that frames the authors' experiences in order to elicit a specific reaction from an imagined audience.

The act of taking an ethnographic approach to using blogs, and other online sources, as data is known as 'netnography' (Kozinets, 2002). The most popular approach to netnography is a passive form in which the researcher takes on the role of observer or reader - known in social media communities as 'lurking'1 (Chan, 2008; Phillips and Broderick, 2014; Mackenzie, 2017). Whilst researchers can remain entirely anonymous and unnoticed, the process also allows contributors to remain anonymous (Hookway, 2008), to the extent that there is arguably no practical reason for blog writers to be aware of the use of their data in research. In other words blog data is available to researchers' to use in just the same way as online journalism or organizational content. The 'backstage' view that blogs allow into personal aspects of people's lives, makes blogs an increasingly attractive data source for social scientists (Chenail, 2011). Nevertheless, whilst there may be strong practical reasons to rely on online data, Bakardijeva and Feeberg (2001) warn of the issue of 'alienation' or the appropriation of blog material for a purpose that was unintended and unforseen by the author. The remainder of this paper seeks to reflect on the way that this 'backstage' and crucially, nonconsensual, view, creates significant ethical issues for the use of blogs as secondary data, and requires considered reflection.

\section{Approach}

The discussion in the remainder of this paper draws on my own experience in a recent research project that explored mothers' experiences of home educating through analysis of autobiographical blogs. This research involved qualitative, thematic analysis of fourteen blogs. These blogs can be considered 'personal', being single authored and largely not-for-profit. However, there was clear evidence that all of the blogs were written for public consumption with most providing ways that the reader could communicate with the author, such as through a comments section. Some of the blogs also acted as a semi-professional enterprise, one of the blogs contained advertising space, whilst two others gave specific information on the sorts of brands they were willing to work with. For some of the blogs, home education was one of many topics covered. For others, it was the primary or exclusive focus. The blogs were all published in English, and were authored by women living predominantly in the UK, US and Australia. Blogs chosen for analysis were identified through a snowballing sampling method, as there are no comprehensive registers of unschooling blogs. Blogs were found through various search engines and websites, and many blogs were found by following links. Ethics approval for this research was obtained through [institution anonymized].

\section{Practical ethics in netnography}

The discussion that follows will focus on some key 'ethical dilemmas' faced during the author's own research. The dilemmas foregrounded here relate to some of the key tenants or at least, the traditions of ethical social science research, that cannot be easily translated to internet-mediated research (Madge, 2007); particularly the issues of consent, authorship and the researcher-participant relationship. Moreover, it will be argued, that traditional rules might not apply. Whilst this discussion focuses on ethical issues in online research, there is likely to be applicability of some of the content of these arguments to non-internet mediated research. Particularly, this broader applicability relates to a critique of the researcher-subject dualism, specifically the automatic assumption of participant vulnerability and the privileging of anonymity and safeguarding over attribution of authorship, when working with participant contributions to research.

\section{Participant consent}

\footnotetext{
${ }^{1}$ Frequently following (reading) online discussing occurring within a particular online space, without contributing.
} 
One key ethical dilemma in the use of online data is the issue of gathering informed consent from content authors (Madge, 2007). Using participant-authored data without their consent flies in the face of contemporary qualitative research ethics that prioritises the need to gather ongoing informed consent from participants (Kara, 2015; Mannay, 2016). However, there is no received wisdom in the social sciences, when the data in question is online (Madge, 2007; Dawson, 2014). Some researchers see gaining written consent from online participants as a central step to the process (Zhang and Hitchcock, 2017; Beninger et al., 2014). However, the approach taken in my own research follows some recent examples, including those of Madge and O'Connor (2006), Roberts (2015) and Pedersen and Lupton (2016) who all used online and publicly-available data without consent. Pedersen and Lupton (2016) explain their decision in the following terms: the data was not elicited nor commented upon by the author, but freely available to the public online; users' identities were not revealed; users were aware that the space in which they were posting was public, and that the content was available to non-members. In the case of the blogs used for my own research, they were entirely open to the public, and as such, there was not deemed to be potential for authors to be unclear about the public audience for their work (Gatson, 2011; Phillips and Broderick, 2014). Despite the public and free availability of the data analysed in my own project, the names of the blogs, authors' names, and some identifying information, were changed (following Hewson, 2003; Pedersen and Lupton, 2016). However, when using internet sources, the use of direct quotations presents a very simple line of identification, due to their easy traceability (Roberts, 2015; Dawson, 2014). For this reason, direct quotations were not used in my own research. Ethically, and academically, this poses a challenge however, given that quotations are the classic approach to evidencing text-based analytic reflections in qualitative research writing.

The concern over the ethics of consent, and the subsequent discussion of protecting participant anonymity, reflects a broader perception of the blog author as 'vulnerable', or at least 'innocent', in much the same way that research participants in traditional face-to-face research have been positioned. This reflects a (perhaps universal) understanding amongst qualitative researchers of the researcher as being in a position of responsibility to both the research community, and the respondents (Illingworth, 2001). It could be argued that this protective, and potentially paternalistic approach is appropriate in research with mothers-who-blog, particularly in the context of the home education blogs explored in my own research. Without significant traffic, these blogs often appear far down on search engines, or not at all, which makes their presence all but invisible, unless you have been invited by the author or perhaps find the blog through one of a handful of other readers. As such, the women writing these blogs might be justified in a presumption that they have a very small audience, perhaps presuming their readership is entirely made up of close family and friends, or indeed, no one at all. This might be likened therefore, to Dawson's 'quiet chat' (2014: 433) and not considered to be legitimate fodder for researchers. There exist a number of dormant blogs on the web, raising a question over whether these mothers should be protected from wider publication and scrutiny, as they might not reasonably foresee their blog holding any interest to outside parties. Nevertheless, these blogs have been written and publicly published and one can assume, that at least at some point, these women did hope for a public readership or recognise the possibility of such (Gatson, 2011; Phillips and Broderick, 2014).

An alternative positioning of mothers who blog is of intelligent actors who engage in writing as a performance that is wittingly undertaken and agential (Madge and O'Connor, 2005). In this characterisation of the author we can imagine the blog as open access data, authored and published material that could be utilised for social science research in the same way as newspaper or magazine articles, editorials or opinion pieces. In this framing of blog authors as cogent produces of social discourse, blogs might be seen as a social commentary and a legitimate piece of secondary data, ripe for qualitative analysis. This is particularly well illustrated through considering 'top' parenting blogs. Firstly, these blogs are often authored by professional or semi-professional writers. Some of these women have journalistic experience and earn an income through writing. Even in cases where blog authors may not be earning income through their blog, the 'top blogs' have considerable reach and influence, with the words produced by these authors potentially having impact in the lives of their readers (Orton-Johnson, 2017). Meanwhile, blogs that are neither professional, nor rated 'top' in their field, may be published to achieve this status at some point, or at least, are being written and published in the hope they may be influential. It therefore seems not only justifiable, but 
perhaps even positive, that blogs should be interrogated by academics, and to some extent held accountable for their content, whether or not the blog author is in agreement with the process.

Moreover, whilst the current focus in academia on social media or blogs heavily emphasises the question of author vulnerability and rights, this narrative is at odds with the professional and legal approach to dealing with public online communication, and with public understanding. In her research with the 'woolly wellbeing' group, Mayne (2017), found that women posting to a closed Facebook group were well aware of the lack of privacy implicit in an online space. Whilst the knitters and crocheters felt supported and safe within the group, they did not presume that it was private. More broadly, public opinion seems to place responsibility for appropriate use of the internet for selfexpression entirely in the hands of the authors (e.g. Sanderson, 2011). For example, eprofessionalism² training informs students and employees of the importance of self-censoring public online communication (Bernhardt et al., 2014; Peck, 2014) - it is well known that many potential employers will conduct searches of online profiles prior to making new hires (Bernhardt et al., 2014; McDonald et al., 2016; Hurrell et al., 2017). Many companies have social media policies that ban employees from publishing words and photographs that undermine the corporate ethos, which might include portraying themselves in a manner deemed unsuitable (Kane et al., 2009; Vaast and Kaganer, 2013; Farnan et al., 2013).

The law also recognises the public as competent actors in their engagement with social media (e.g. in England the Defamation Act 2013; Copyright Designs and Patents Act, 1988; the Racial and Religious Hatred Act 2006 can all be applied to individuals' communications on social media). This is illustrated through recent high profile convictions of members of the public as a result of Facebook posts. On $13^{\text {th }}$ July 2017 Rhodri Phillips, an English aristocrat, was jailed for 12 weeks following racist Facebook posts and placing a bounty on the head of Gina Miller, a high profile anti-Brexit campaigner. In Zurich earlier this year (2017), a number of contributors to a Facebook attack on the animal-rights activist Erwin Kessler were sued for anti-Semitic and racist comments, including one man who simply 'liked' some of the existing comments. In an Australian example, an Australian Football League fan was charged by police for racist remarks addressed at an aboriginal football player in an online posting. Beyond responsibility for online publishing, there is increasing expectation that public users of the internet ought to be fully aware of the broad implications of their use and the visibility of their commentary. In a recent case in San Jose, California, District Judge Edward Davila threw out a case against Facebook's alleged tracking of internet users, arguing that the complainant should have known to use an opt-out tool or an 'incognito' mode for browsing the internet.

\section{Using blog data as least ethically challenging}

Research that involves interaction with participants and the generation of data for the researcher, or the personal gifting of privately held information to the researcher, involves a contract. As part of this participation agreement, typically referred to as informed consent, the researcher pledges to attempt to protect the participant from harm. This protection from harm - whether in the form of avoiding coercion to participate, or protection of participants' identities, is not always infallible. In my own research papers I have reflected on children being 'forced' into participation (REFERENCE TO AUTHOR'S WORK), whilst Sparks et al. (2016) discuss the ramifications of errors that lead to a breach in the participants' anonymity when using digital technology in research. These situations, unintentional accidents of the research process, are only created due to the research requiring active and purposeful contributions from participants. By contrast, research that does not require the production or disclosure of any particular data, minimises the potential harm. In the case of online research, the researcher is not encouraging the author to create data for the purposes of the project. Increased risk of harm to participants concerns solely the potential for increased traffic to the blog, or an alternative interpretation of blog content.

\footnotetext{
${ }^{2}$ The craft of developing a professional persona online.
} 
Using blogs allows the researcher to access data that has been produced by the author, usually for a specific purpose that is articulated by the author. Writing about their experiences allows blog authors to construct their own meanings from these phenomena, and to prioritise and categorise their own narratives (Keller, 2012; Friedman, 2013). This is in contrast to researcher-led activities in which a participant will construct responses that relate to questions concerning themes that are prioritised and ordered by the researcher. Bloggers have freedom to edit and re-edit their written communication until they are sure that it reflects the narrative that they wish to share. As such, it might be argued that the use of written blogs could be a more accurate reflection of participants' priorities and emotions. It could also be argued that blogs have the potential to be a more polished or less 'truthful' reflection of their experience, reflecting broader concerns about the imaginary communicated online (Bartholomew et al., 2012; Jane and Dworkin, 2014; Dawson, 2016). However, allowing a bloggers' voice to be used in the manner in which they are most comfortable must be more ethical than putting participants on the spot to produce more naturalistic responses, or relaxing participants into disclosure in a friendly one-to-one research setting, meanwhile knowing that you intend to analyse and present the research data in publications to a broad audience.

\section{Concluding remarks}

My advocacy for the use of online, publicly-available, data is underpinned by a philosophical approach that the use of this data, rather than putting a vulnerable person at risk of harm, is in fact demonstrating the utmost respect for the writer of the blog as 'author'. As such, my conclusions is that researchers should approach blogs with a presumption of accountability. That is to say that researchers should presume a blog author is a cogent actor who has purposefully published content, and acknowledge them as such in discussion and potential analysis of the blog authors' commentary. Using this approach, blogs might be cited in the same way as online newspaper columns, complete with names, data of access, and online location for reference. A researcher might chose to inform a blogger of any academic publications relating to the blog, but would not be obliged to seek informed consent, or approval prior to publication. It is important to underscore here, that a presumption of accountability is not synonymous with a universal rule of accountability, and that researcher discretion and judgment is certainly required when considering whether there might be a duty to safeguard the blog author, for example if the author is a child, and how to approach the use of public comments that might accompany a published blog.

My conclusion moves beyond Hine (2005) in not only reflecting on the incapacity of institutionalised ethics procedures to adequately contend with online research spaces, but wondering if online research in fact emphasises the flaws in bureaucratic ethical evaluation in all qualitative research spaces. Incorporating the words of authors, and potentially, giving them credit for their contribution to the research, simply involves the treatment of blog authors in a manner in which we as research students or professionals would wish to be treated, and the way in which we would expect professional writers and journalists to be treated. To this end, the paper follows Madge (2007) in her critique of 'ethical colonialism' that she considers implicit in a researcher-subject dualism that is typically written into academic bureaucracy. What is key here, if we are to begin to question the requirement for consent and anonymity, and even to credit the authors of the internet-based sources that we use, is the necessity to ground our work in a personal ethics of care (Capurro and Pingal, 2002) that seeks to do no harm, and better, to improve the lived experiences of those in positions of less power. The ways in which we might do this are, of course, as many and varied as the potential researchers and participants themselves.

\section{Acknowledgements}

With many thanks to Kim O'Sullivan for valuable feedback on a draft of this paper.

\section{References}

Bartholomew MK, Schoppe-Sullivan M, Glassman M, Kamp Dush CM, Sullivan JM.2012 New parents' Facebook use at the transition to parenthood Family Relations 613 455-469 
Bakardjieva M, and Feenberg, A 2001 Involving the virtual subject Ethics and Information Technology 2 233-40

Dawson P2014"Our anonymous online research participants are not always anonymous: Is this a problem?" British Journal of Educational Technology 453 428-37

Beninger K A, Fry N, Jago H,. Lepps L, Nass, and Silvester H 2014 Research using social media: Users' views. London, UK: NatCen (National Centre for Social Research).

Bernhardt M, Alber J, Gold RS 2014 A social media primer for professionals: digital do's and don'ts Health Promot Pract 152 168-172

Capurro R and C Pingel 2002 Ethical issues of online communication research Ethics and Information Technology 4 189-94

Chan A 2008 'Life in Happy Land': using virtual space and doing motherhood in Hong Kong Gender, Place and Culture 152 169-188

Chenail R 2011 Qualitative researchers in the blogosphere: Using blogs as diaries and data The Qualitative Report 16 $1249-254$

Choi P, Henshaw C, Baker S, and Tree J 2005 Supermum, superwife, supereverything: performing femininity in the transition to motherhood Journal of reproductive and infant psychology $232167-180$

Cline RJ and Hayes KM 2001 Consumer health seeking on the Internet: State of the art Health Education Research 16 $6671-692$

Crooks V 2006 'I go on the internet: I always, you know, chck to see what's new': chronically ill women's use of online health information to shape and inform doctor-patient interactions in the space of care provision ACME: An International E-Journal for Critical Geographies 5 50-69

Davidson J 2008 Autistic culture online: virtual communication and cultural expression on the spectrum Social and Cultural Geography, 9, 791-806

Dawson P 2014 “Our anonymous online research participants are not always anonymous: Is this a problem?" British Journal of Educational Technology 453 428-37

Dawson M 2016 Parenting in a Fakebook World: How Soccial Media is Affecting Your Parenting, online, available at: www.parent.com/parenting/better-parenting/style/how-social-media-is-affecting-your-parenting/

DeChoudhury M, Gamon M, Counts S. and Horvitz E 2013 Predicting depression via social media Proceedings of the Seventh International AAAI Conference on Weblogs and Social Media, Boston, MA.

Dwyer, C., and Davies, G., (2010), Qualitative methods III: animating archives, artful interventions and online environments, Progress in Human Geography, 34, 1, 88-97

Farnan JM, Snyder Sulmasy L, Worster BK, Chaudhry HJ, Rhyne JA, Arora VM 2013 Online medical professionalism: patient and public relationships, policy statement from the American College of Physicians and Federation of State Medical Boards Ann Intern Med 1588 620-7

Friedman M 2013 Mommyblogs and the changing face of motherhood Toronto: University of Toronto Press

Gatson SN 2011 The methods, politics, and ethics of representation in online ethnography The SAGE Handbook of Qualitative Research 4 513-527

Gray D 2009 Doing research in the real world (2nd ed.) London: SAGE Publications

Hewson C, Yule P, Laurent D and Vogel C 2003 Internet research methods London: Sage

Hine C 2015 Ethnography for the internet: Embedded, embodied and everyday London, UK: Bloomsbury Publishing

Hookway N 2008 'Entering the blogosphere': Some strategies for using blogs in social research Qualitative Research 81 91-113

Hurrell S A, Scholarios D and Richards J 2017 'The kids are alert': Generation Y responses to employer use and monitoring of social networking sites New Technology, Work and Employment, 32: 64-83

Illingworth N 2001 'The Internet Matters: Exploring the Use of the Internet as a Research Tool Sociological Research Online 62 1-17

Jang J and Dworkin J 2014 Does social network site use matter for mothers? Implications for bonding and bridging capital Computers in Human Behavioiur 35 489-495 
Johnson SA 2015 'Intimate mothering publics': comparing face-to-face support groups and Internet use for women seeking information and advice in the transition to first-time motherhood Culture, Health and Sexuality: An International Journal for Research, Intervention and Care 172 237-251

Kane G C, Fichman R G, Gallaugher J and Glaser J 2009 Community relations 2.0 Harvard Business Review 45-50 Kara H 2015 Creative Research Methods in the Social Sciences: A practical guide Bristol: Policy Press

Keller JM 2012 Virtual feminisms: Girls' blogging communities, feminist activism, and participatory politics, Information, Communication and Society 15 429-447

Kinsley S 2014 The matter of 'virtual' geographies Progress in Human Geography 38 364-384

Kitchen R 1998 Towards geographies of cyberspace Progress in Human Geography 22 385-406

Kozinets R 2002 The field behind the screen: Using Netnography for marketing research in online communities Journal of Marketing Research $39161-72$

Laird S 2012 The Rise of the Mommy Blogger [Infographic], online, accessed 11.08.2017, available at:

www.mashable.com/2012/05/08/mommy-blogger-infographic

Longhurst R 2009 YouTube: A new space for birth? Feminist Review 93 26-63

Lopez LK 2009 The radical act of 'mummy blogging', redefining motherhood through the blogosphere New Media and Society 11 729-727

Mannay D 2016 Visual, Narrative and Creative Research Methods: Application, reflection, ethics Abingdon:

Routledge

Mackenzie J 2017 'Can we have a child exchange?' Constructing and subverting the 'good mother' through play in Mumsnet Talk Discourse and Society 283 296-312

Madge C 2007 Developing a geographers' agenda for online research ethics Progress in Human Geography 315 654674

Madge C and O'Connor H 2006 Parenting gone wired: empowerment of new mothers on the internet? Social and Cultural Geography 72 199-220

Mayne A 2017 Virtually Ethical: Ethnographic challenges in researching textile crafters online in Daniels J Et al (eds) Digital Sociologies Bristol: Policy Press 59-74

McDaniel BT, CoyneSM, Holmes EK 2012 New Mother and Media Use: Associations Between Blogging, Social Networking, and Maternal Well-Being Maternal Child Health Journal 16 1509-1517

McDonald P Thompson P and O'Connor P 2016 Profiling employees online: shifting public-private boundaries in organisational life Human Resource Management Journal 26 541-556

McGannon KR, McMahon J, and Gonsalves, C 2017 Mother running in the blogosphere: A discursive psychological analysis of online recreational athlete identities Psychology of Sport and exercise 28 125-135

Orton-Johnson K 2017 Mummy blogs and representations of motherhood: 'bad mummies' and their readers Social Media and Society 1-10

Peck JL 2014. Social media in nursing education: responsible integration for meaningful use. J Nurs Educ.19:1-6 Pedersen S and Lupton D 2016 'What are you feeling right now?'communities of maternal feeling on Mumsnet. Emotion, Space and Society available online

Pedersen S and Smithson J 2013 Mothers with attitude-How the Mumsnet parenting forum offers space for new forms of femininity to emerge online Women's Studies International Forum 38 97-106

Philips N and Broderick A 2014 Has Mumsnet changed me? SNS influence on identity adaptation and consumption, Journal of Marketing Management 30 9-10 1039-1057

Sanderson J 2011 To Tweet or Not To Tweet: Exploring Division 1 Athletic Departments' Social Media Policies International Journal of Sport Communication 44 492-

Snee H 2013 Making Ethical Decisions in an Online Context: Reflections on Using Blogs to Explore Narratives of Experience Methodological Innovations 82 52-67

Sparks H, Collins FL, and Kearns, R 2016 Reflecting on the risks and ethical dilemmas of digital research Geoforum 77 40-46

Thomson L and Cupples J 2008 Seen and not heard: Text messaging and digital society Social and Cultural Geography 9 95-108 
Valentine G 2006 Globalising intimacy: The role of information and communication technologies in maintaining and creating relationships Women's Studies Quarterly 34 365-393

Vaast, E. and Kaganer, E 2013 Social media affordances and governance in the workplace: An examination of organizational policies. J Comput-Mediat Comm 19: 78-101

Zhang Y and Hitchcock M J 2017 The Chinese female tourist gaze: a netnography of young women's blogs on Macao Current Issues in Tourism 203 315-330 